\title{
The Resuming Insulation Discharge Failure and Conductor Overheating Failure Judgment Method of $\mathrm{SF}_{6}$ Electrical Equipment
}

\author{
Xiansheng ZHUANG, Zhi LI, Chengji HUANG \\ Electric Power Research Institute of Guangdong Power Grid Corporation, Guangzhou, China \\ Email: zhuangxs@163.com
}

\begin{abstract}
Through the long time track examination and disintegration to $\mathrm{SF}_{6}$ circuit breaker, we obtain the massive monitor data and massive pictures. The criteria of resuming insulation discharge failure conforming to $\mathrm{C}_{\mathrm{SO} 2} / \mathrm{C}_{\mathrm{H} 2 \mathrm{~S}}>7$, is quite broad to $\mathrm{SO}_{2}$ and the $\mathrm{H}_{2} \mathrm{~S}$ concentration permission. Even if it reaches $100 \mu \mathrm{L} / \mathrm{L}$, it will not be in danger immediately to the safe operation of equipment. We may plan, arrange, and overhaul calmly. When obtaining the bare conductor overheating failure, it has not involved the resuming insulation. We may use the resuming insulation discharge failure criterion.
\end{abstract}

Keywords: $\mathrm{SF}_{6}$; conductor, discharge, overheated, failure, method

\section{1. 引 言}

随着 $\mathrm{SF}_{6}$ 电气设备的大量使用, 设备内部各种放 电和过热故障时有发生, 严重影响 $\mathrm{SF}_{6}$ 电气设备的安 全、可靠运行。目前，在国际上，还没有关于 $\mathrm{SF}_{6}$ 电 气设备内部放电和导体过热故障的判断标准, IEC60480 $0^{[1]}$ 只是给出了运行中 $\mathrm{SF}_{6}$ 电气设备 $\mathrm{SF}_{6}$ 气体 中分解物的最大限值。在国内, 《The examination and judgment of $\mathrm{SF}_{6}$ Electrical Equipment Discharge Failure》 ${ }^{[2]}$ 给出了有关 $\mathrm{SF}_{6}$ 电气设备内部放电判断依据。 本文通过对有故障设备进行长时间的跟踪监测, 最后 对设备进行检修解体, 获得大量的监测数据和图片等 资料, 总结出 $\mathrm{SF}_{6}$ 电气设备自恢复绝缘放电故障和导 体过热故障的判断依据, 对 $\mathrm{SF}_{6}$ 电气设备的放电故障 判断具有重要意义。

\section{2. 设备概括}

某公司 $110 \mathrm{kV}$ 六氟化硫组合电器, 设备参数如下:

设备型号: QF1-110, 额定电压 : $110 \mathrm{kV}$, 额定电流: $1250 \mathrm{~A}$, 额定开断电流 : $31.5 \mathrm{kA}$, 制造日期 : 1988 年。

\section{3. 设备运行历史}

该设备 1988 年投厂后, 2001 年 12 月 19 日发生 \# 1 主变 110 高压侧 B 相户外侧套管断裂掉下短路事
故。

\section{1. 事故单元概况}

事故前运行工况 : 黄石甲线经 127 开关送 $110 \mathrm{KV}$ 乙母线带1\#、2\#、3\#主变运行, 黄石乙线经128开关送 $110 \mathrm{KV}$ 甲母线空载热备用。

$6 \mathrm{KV}$ 系统：6023 分段开关“合” (上主 6KVV、

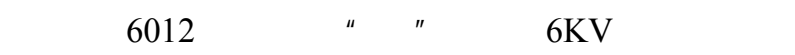
6015 母联开关“合” (上主 $6 \mathrm{KV}$ I、V段)；6025 母 联开关“分”作热备用状态；6034 分段开关“合”（上 主 $6 \mathrm{KV}$ III、IV段)；6046 母联开关 合” (上主 KV IV、 VI段)；6036母联开关“分”作热备用状态。

\section{2. 事故经过}

2001 年 12 月 19 日 8:12 时, 1 \#主变高压侧 B 相 穿墙套管户外侧突然断裂掉下, $110 \mathrm{KV}$ 乙对地短路。 1 \#主变保护动作跳主变低压侧开关、高压侧 101 开关 拒动; 2 \#主变“零序过流” 保护动作跳主变两侧开关 ; 3 \#主变“ 复合电压闭锁过流” 保护动作跳主变两侧开 关 ; 4\#主变“复合电压闭锁过流”保护动作跳主变两 侧开关及 6034、6046 母联开关。厂电力系统与市网解 列运行，同时黄埔电厂 127 开关也跳闸，厂主 $6 \mathrm{KV}$ 系 统分成三个独立系统运行。8:14 时 2\#发电机“复合电 压闭锁过流”保护动作跳闸，热 116 开关保护跳闸，乙 
烯单回路供电; 黄埔电厂127 开关重合闸时，101 开 关保护动作跳开。

\section{3. 事故处理过程及恢复情况}

12 月 29 日更换 1 \#主变高压侧三个穿墙套管, 12 月 30 日 16:50 时 $1 \#$ 主变送电并网运行。

\section{4. 事故原因分析}

\subsection{1. 主要原因}

\#1主变高压侧B相穿墙套管户外侧突然断裂, 造 成 $110 \mathrm{kV}$ 乙 $\mathrm{B}$ 相对地短路。套管断裂的原因是套管本 身质量存在缺陷:套管瓷体有未烧透的夹层; 固定套管 的法兰存在旧裂纹, 内应力作用使套管断裂, 断裂后 放电，断口靠乙母侧的套管内壁有严重的放电烧弧痕 迹，而另一侧套管内壁则没有。

\subsection{2. 间接原因}

1 \#主变第一次差动保护动作后, 101 开关拒动, 未能及时切断故障点。使事故影响范围扩大。

101 开关拒动是由于操作机构的液压油受低温天 气影响, 油粘度大, 机构动作迟缓。

\section{4. 故障的发现、判断和追踪监测情况}

\section{1. 放电故障的发现}

2008 年 2 月 26 日, 应某公司要求, 对某公司所 属的 $\mathrm{SF}_{6}$ 电气设备进行水分、二氧化硫和硫化氢的预 防性试验, 全部测试六氟化硫组合电器 252 个气室

$(110 \mathrm{kV}$ 加氢联合变 23 气室、热电站 32 气室、热电 站升压变 8 气室、乙烯总降 14 气室、动力二站 68 气 室、炼化站 107 气室), 除热电站 $110 k V G I S ＃ 1$ 主变 101 开关以外, 其他气室未发现异常放电缺陷。热电 站 $110 \mathrm{kVGIS}$ \# 1 主变 101 开关气室 $\mathrm{SF}_{6}$ 气体中, 二氧 化硫浓度大于 $146 \mu \mathrm{L} / \mathrm{L}$, 硫化氢浓度 $26.14 \mu \mathrm{L} / \mathrm{L}$, 判 断该设备存在异常放电故障, 建议有条件尽快检修, 测试结果见表 1 。

2008 年 3 月 5 日应厂方要求, 分相测试二氧化硫 和硫化氢, 并取样测试气体分解产物。测试结果判断 故障点在 B 相, 其他两相是否存在放电缺陷需要进一 步确认。

从测试结果看, 虽然 B 相二氧化硫浓度大于 146 $\mu \mathrm{L} / \mathrm{L}$, 超过仪器检测最高限, 可以判断 B 相内部存 在或曾经发生过放电故障，存在放电缺陷; 从二氧化
硫和硫化氢浓度比值大于 7 (从 2008 年 2 月 26 日和 2008 年 3 月 5 日测试结果综合判断), 可以判断放电 缺陷属自恢复绝缘放电故障, 放电部位和放电通道主 要涉及自恢复绝缘。当时判断对设备的运行没有即时 危险性。

\section{2. 追踪测试}

由于通过检测六氟化硫电气设备中二氧化硫和硫 化氢组分浓度, 判断设备是否存在放电缺陷以及放电 的类型、涉及的绝缘种类 (自恢复绝缘或非自恢复绝 缘)，在目前还是新生事物，还没有相关的规程和标准。 厂方对我们的判断有所保留，仍然不相信该设备存在 异常放电故障。为了便于监测，2008 年 3 月 5 日对 101 开关进行更换 $\mathrm{SF}_{6}$ 气体工作。24 小时后，3 月 6 日进 行现场测试和取样测试。2008 年 3 月 6 日 2008 年 10 月 23 日追踪测试结果汇总见表 1 。

10 月 23 日检测结果, 二氧化硫已经超过仪器检 测最高限。2008 年 12 月 1 日再次对 101 开关进行更 换 SF6 气体处理，并把设备停运 (处于冷备用状态)。 并跟踪检测二氧化硫和硫化氢（此时二氧化硫和硫化 氢为吸附剂释放结果, 非由设备放电产生)，与以前的 检测结果对比, 以判断二氧化硫和硫化氢增长是否由 设备内部放电产生。

101 开关冷备用状态跟踪测试进行了 4 个月，检 测结果汇总见表 1 。

\section{3. 更换 101 开关}

通过 101 开关的运行和停运测试结果对比，可以 判断 101 断路器存在放电故障。厂方联系北京开关厂, 于 2009 年 5 月 1 日对 101 开关进行更换检修工作。更 换后的检测结果见表 1 。从表 1 可以看出, 101 开关更 换检修以后，两次检测结果都未检出二氧化硫和硫化 氢，说明故障消除。

\section{101 开关解体情况和故障分析}

\section{1. 固体绝缘完好, 满足断路器运行绝缘要求}

1) 支撑绝缘筒内部和外部玻璃纤维环氧树脂绝 缘件都没有损伤和破坏。

2) 支撑绝缘杆 (6根) (玻璃纤维环氧树脂绝缘件) 表面未发现任何损伤和破坏。

3) 上下出线绝缘子 (绝缘盘) (环氧树脂绝缘件) 表面未发现任何损伤和破坏，虽然上绝缘子表面粘附 
表1. 110kVGIS \#1主变101开关追踪测试结果汇总

Table 1. 101 breaker sulfur hexafluoride gas component test result

\begin{tabular}{|c|c|c|c|c|c|c|c|c|c|}
\hline 测试项目 item & 空气 & 四氟化碳 & 六氟化硫 & 二氧化碳 & 氟化亚硫酰 & 氟化硫酰 & 十氟化二硫酰 & 二氧化硫 & 硫化氢 \\
\hline \multirow{2}{*}{ 测试日期 date } & & $\mathrm{CF}_{4}$ & $\mathrm{SF}_{6}$ & $\mathrm{CO}_{2}$ & $\mathrm{SOF}_{2}$ & $\mathrm{~S}_{2} \mathrm{OF}_{10}$ & $\mathrm{~S}_{2} \mathrm{OF}_{10}$ & $\mathrm{SO}_{2}$ & $\mathrm{H}_{2} \mathrm{~S}$ \\
\hline & $\% \mathrm{~m} / \mathrm{m}$ & $\% \mathrm{~m} / \mathrm{m}$ & $\% \mathrm{~m} / \mathrm{m}$ & $\% \mathrm{~m} / \mathrm{m}$ & $\% \mathrm{~m} / \mathrm{m}$ & $\% \mathrm{~m} / \mathrm{m}$ & $\% \mathrm{~m} / \mathrm{m}$ & $\mu \mathrm{L} / \mathrm{L}$ & $\mu \mathrm{L} / \mathrm{L}$ \\
\hline 2008年 2月 26日 & - & - & - & - & - & - & - & $>146$ & 26.14 \\
\hline 2008年 3月 5日 A & 0.0246 & 0.1325 & 99.79 & 0.0093 & 0.0016 & 0.029 & 0.0132 & 6.86 & 0.43 \\
\hline 2008年 3月 5日 B & 0.0268 & 0.2335 & 99.70 & 0.0169 & 0.0018 & 0.011 & 0.0078 & $>146$ & 59.14 \\
\hline 2008年 3月 5日 C & 0.0292 & 0.1227 & 99.81 & 0.0083 & 0.0017 & 0.016 & 0.0127 & 14.71 & 1.00 \\
\hline 2008年 3月 6 日 & 0.0070 & 0.0045 & 99.989 & 未检出 & 未检出 & 未检出 & 未检出 & 6.29 & 0.00 \\
\hline 2008年 3月 12日 & 0.0011 & 0.0031 & 99.983 & 未检出 & 0.0131 & 未检出 & 未检出 & 23.86 & 1.00 \\
\hline 2008年 4月 14日 & 0.0012 & 0.0020 & 99.997 & 未检出 & 未检出 & 未检出 & 未检出 & 45.29 & 5.16 \\
\hline 2008年 5月 27日 & 0.0012 & 0.0036 & 99.995 & 未检出 & 未检出 & 未检出 & 未检出 & 60.43 & 8.86 \\
\hline 2008年 7月 29日 & 0.0059 & 0.0059 & 99.971 & 未检出 & 0.0042 & 未检出 & 0.0131 & 79.00 & 10.14 \\
\hline 2008年 8月 26日 & 0.0020 & 0.0036 & 99.984 & 未检出 & 0.0008 & 未检出 & 0.0093 & 96.43 & 13.71 \\
\hline 2008年 9月 24日 & 0.0132 & 0.0010 & 99.986 & 未检出 & 0.0042 & 未检出 & 0.0131 & 100.6 & 14.14 \\
\hline 2008年 10月 23日 & - & - & - & - & - & - & - & $>146$ & 25.14 \\
\hline 2008年 12月 2日 & - & - & - & - & - & - & - & 1.74 & 0.09 \\
\hline 2009年 1月 6日 & - & - & - & - & - & - & - & 3.34 & 0.24 \\
\hline 2009年 2月 10日 & - & - & - & - & - & - & - & 5.44 & 0.94 \\
\hline 2009年 3月 13日 & - & - & - & - & - & - & - & 7.61 & 1.01 \\
\hline 2009年 5月 9日 & - & - & - & - & - & - & - & 未检出 & 未检出 \\
\hline 2009年 5月 18日 & - & - & - & - & - & - & - & 未检出 & 未检出 \\
\hline
\end{tabular}

的粉尘有放电痕迹, 但粉尘擦除后发现粉尘表面的放 电痕迹并未伤及绝缘子。

4) 操作传动绝缘杆 (玻璃纤维环氧树脂绝缘件) 表面未发现任何损伤和破坏。

\section{2. 导体电流路径有些损伤, 但基本仍能满足开 关运行要求}

1) 上下出线导体触头有受电弧灼伤过热痕迹。触 头触子接触位置保持良好。

2) 动触头受电弧灼伤过热, 损伤较严重, 多数触 子仍能与静触头保持良好接触, 满足额定电流通过要 求。动触头触子与上下静触头接触位置明显接触划痕 不均匀。说明动触头触子由于受电弧高温灼伤, 接触
压紧力受影响。

3) 上下静触头虽然受电弧灼伤过热较严重, 但与 动触头触子的接触电阻仍能满足额定电流通过要求。 下静触头石墨喷嘴受电弧烧蚀严重。由于动触头触子 接触压紧力不均匀且上下静触头受电弧高温灼伤、过 热，表面氧化严重，运行电流大时容易产生过热。

5.3. 自能压气灭弧室损伤严重, 严重影响灭弧室 的吹弧能力, 基本丧失对开断短路电流的灭 弧能力

1) 压气室上密封盖脱落, 并且烧蚀严重, 基本丧 失自能高压吹弧功能。

压气室外筒为玻璃丝编织绝缘件 ; 压气室密封盖 
材料为聚四氟乙烯。两者采用胶粘固定在一起。一方 面在断路器分闸过程中跟随动触头向下移动, 与固定 活塞作用使压气室空间缩小从而产生高压气体满足吹 弧要求; 另一方面在电弧的高温作用下, 压气室中气 体产生吹弧需要的高压。

由于密封盖松脱, 使压气室内部不能保持产生高 压气体, 虽然根据设计要求, 仍能满足开断额定电流 以下的操作, 但已严重影响对短路大电流的灭弧能力, 有可能产生严重后果

2) 根据断路器内部情况、灭弧室损坏情况可以判 断断路器曾经发生未能按断路器功能要求及时灭弧, 从而产生如下几方面的损坏或损伤 :

a) 压气室内部空间由于未能及时灭弧, 在电弧高 温作用下, 压气室外筒与密封盖之间的胶粘融化失去 粘接能力, 在压气室内高压气体作用下而松脱。

b) 由于较长时间的燃弧，使密封盖烧蚀严重；

c) 下静触头石墨喷嘴烧蚀严重 ;

d) 动触头触子受损, 影响接触压紧力 ;

e) 压气室外筒受热, 温度升高, 表面的胶漆熔融 或滴落。

f) 从石墨喷嘴烧蚀位置和压气室密封盖烧蚀位 置基本在同一垂直线上, 可以判断电弧热气流从该位 置上升喷出压气室外。

从石墨烧蚀喷嘴、动触头的水平相对位置, 可以 判断断路器已经产生开断动作, 动触头与上静触头之 间在刚要脱离瞬间, 即已开始燃弧, 由于断路器操作 机构原因或是由于电磁力的作用, 造成断路器的开断 动作没能继续, 也就没有进入吹弧阶段, 压气室内空 间在高温电弧作用下, 气体压力极高, 电弧热气流冲 开密封盖外泄。燃弧时间持续到其他保护跳闸，切断 母线和主变电流而熄灭。

g) 石墨喷嘴烧蚀产生的分解物主要为四氟化碳 $\left(\mathrm{CF}_{4}\right)$ ，在 2008 年 3 月 5 日以前的测试数据中，四 氟化碳浓度很高, 见表 1 , A 相 $0.1325 \% \mathrm{~m} / \mathrm{m}, \mathrm{B}$ 相 $0.2335 \% \mathrm{~m} / \mathrm{m}, \mathrm{C}$ 相 $0.1227 \% \mathrm{~m} / \mathrm{m}$ 。

在 101 开关更换 $\mathrm{SF}_{6}$ 气体以后, 从 2008 年 3 月 6 日 2008 年 10 月 23 日, 几个月的跟踪测试, 四氟化 碳浓度都变化不大, 基本在 $0.0060 \% \mathrm{~m} / \mathrm{m}$ 以下。说明 在 2008 年 3 月 6 日 2008 年 10 月 23 日的时间范围 内, 未曾发生过未能按断路器功能要求及时灭弧的情 况, 即在该时间段内, 断路器没有发生大电流、长时 间燃弧的情况。
5.4. 由于高温粉尘等材料落下, 造成断路器底座 油漆熔融或松脱。

5.5. 断路器的正常开断、闭合和异常开断、闭合

断路器的开断和闭合操作燃弧可以分为正常燃弧 和异常燃弧，这些都是属于有目的性的操作，并且燃 弧一般都是局限在灭弧室内部, 不属于断路器异常放 电范围。

\subsection{1. 断路器的正常开断和闭合}

断路器正常开断燃弧和灭弧过程, 由于灭弧室的 特殊设计, 电离、离子化、分解的 $\mathrm{SF}_{6}$ 气体在该过程结 束后一般都能很好的复合。 $\mathrm{SF}_{6}$ 气体由于断路器正常开 断和闭合过程产生的分解产物极少, 设备内部的吸附 剂可以慢慢把它吸附。根据多年的检测结果显示, 正 常开断和闭合的断路器, 即使运行十几、二十年的断 路器, 其内部的 $\mathrm{SF}_{6}$ 气体都检测不到二氧化硫和硫化 氢, 这是可以利用检测二氧化硫和硫化氢作为判断断 路器内部异常放电缺陷的首要前提条件。

\subsection{2. 断路器的异常开断和闭合}

断路器异常开断和闭合, 分以下几种情况 :

1) 断路器开断、闭合过程燃弧, 但由于操作机构 或其他原因造成断路器不能延续开断、闭合动作, 需 要其他保护动作，切断电源电流而灭弧；

2) 断路器在开断、闭合过程不能有效灭弧, 需要 其他保护动作，切断电源电流而灭弧；

3) 断路器开断、闭合额定短路电流以上的电流。

断路器异常开断和闭合, 有如下特点:

1) 由于都是有目的性的操作, 异常现象当时就能 发现。

2) 都会对灭弧室动、静触头, 吹弧喷嘴, 压气室 等造成不同程度的烧蚀或其他损害 ;

3) 都会产生不同浓度的二氧化硫和硫化氢等分 解产物，浓度相对正常操作状态大得多。

4) 发现异常现象以后, 及时进行分析、检测, 就 能判断断路器是否受到损害, 断路器功能是否仍然有 效, 及时采取必要的措施，包括继续追踪监测、检修 等, 就能确保设备的安全运行。

如本文中 101 开关情况, 如果及早进行分解产物 二氧化硫和硫化氢监测，就能及时发现 101 开关的缺 陷。幸好 101 开关灭弧室受到损害以后, 未曾开断短 路大电流。 


\section{6. 断路器内部异常放电和过热缺陷}

设备内部异常放电缺陷不包括以上断路器正常、 异常开断和闭合情况。虽然断路器异常开断和闭合, 电弧的能量异常大或不受控, 与断路器正常开断、闭 合相比, 会产生大量的 $\mathrm{SF}_{6}$ 分解产物。这些属于设备操 作异常，这种操作异常事后是可知的。

设备内部异常放电缺陷是无目的性的、未知的、 由于某些原因而在设备内部悄然进行的现象。由于是 未知的, 未能及时发现, 特别是非自恢复绝缘的放电, 对设备安全造成较大威胁。所以, 需要象设备预防性 试验一样, 定期进行分解产物二氧化硫和硫化氢的检 测, 才能尽早发现设备内部的异常放电缺陷, 采取必 要的措施, 确保设备安全运行。

根据对 101 开关的一年多的跟踪监测，判定 101 开关内部存在异常放电缺陷。并且根据二氧化硫和硫 化氢比值大于 7 判定, 异常放电主要涉及自恢复绝缘。

根据 101 开关解体情况, 101 开关内部异常放电 和过热缺陷并存, 要分清楚放电痕迹是在追踪测试期 间还是以前发生有些困难，根据解体情况判断，101 开关在追踪监测期间，放电和过热并存。有以下几方 面 :

\subsection{1. 局部放电 (电晕放电)}

由于断路器开断大电流失败以后, 燃弧时间过长, 灭弧室内部气体压力极高。高压气体几乎像产生爆炸 似地从灭弧室外泄，气体夹带大量粉尘。电弧熄灭以 后, 粉尘慢慢沉积在设备内部表面。

一方面由于灭弧室压气盖松脱, 另一方面压气盖 一边烧蚀严重。在合闸状态下, 压气盖处于上静触头 位置, 在上静触头周围产生严重不均匀电场。由于灭 弧室内部受损严重, 下静触头的石墨喷嘴严重烧蚀产 生凹坑等, 造成喷嘴附近场强畸变, 产生严重极不均 匀电场。灭弧室的严重破坏, 造成内部极不均匀电场, 在高场强区域产生局部放电。

另外, 上下绝缘盘的连接触头导体表面也产生电 晕放电。

\subsection{2. 裸导体过热}

101 开关上下静触头材料为钢合金，与动触头触 子接触表面为铜铇合金，端部喷口封接石墨作为灭弧 喷嘴。

101 开关动触头主要材料也是钢合金, 触子为铜合
金和铜铇合金。

101开关由于经历过开断短路大电流失败，对灭弧 室内部造成极大的损害。特别是动触头触子及其电流 路径材料的损害, 接触压紧力受影响，一方面造成触 子与上下静触头接触不均匀, 这从触头触子与静触头 的接触移动划痕可以看出来。

静触头表面氧化严重，氧化层厚度大，影响接触 电阻。在运行电流较大时，容易产生导体过热。

一方面由于灭弧室压气盖松脱, 另一方面压气盖 一边烧蚀严重。在合闸状态下，压气盖处于上静触头 位置。在上静触头周围产生严重不均匀电场，引起局 部放电。

101 断路器的导体过热（没有涉及非自恢复绝缘 (固体绝缘)），使六氟化硫气体分解，产生的特征组 分浓度含量特征与裸金属放电特征基本一致，二氧化 硫和硫化氢比值判定标准一样适用。

\subsection{3. 底座粉末主要成分}

抽取 101开关B相底座粉末进行发射光谱测试，主 要金属成分为铜和铇。基本符合铜铇触头合金的成分 比例，说明设备中触头烧蚀严重。

101 开关 B 相底座粉末粉尘测试非金属成分结 果：S3.52\%，C29.64\%。石墨为非金属碳的一种结构 形式; 聚四氟乙烯是碳的氟化物。从粉末测试结构含 碳 $29.64 \%$ ，石墨喷口和聚四氟乙烯密封盖烧蚀严重。

\section{6. 结论}

1) 检测 $\mathrm{SF}_{6}$ 电气设备 $\mathrm{SF}_{6}$ 气体中 $\mathrm{SO}_{2}$ 和 $\mathrm{H}_{2} \mathrm{~S}$ 的浓 度判断 $\mathrm{SF}_{6}$ 电气设备内部放电和过热故障是有效的手 段。结合预防性试验周期，进行 $\mathrm{SF}_{6}$ 电气设备 $\mathrm{SF}_{6}$ 气 体中 $\mathrm{SO}_{2}$ 和 $\mathrm{H}_{2} \mathrm{~S}$ 的定期检测，可以及早发现 $\mathrm{SF}_{6}$ 电气 设备内部的异常放电和过热故障。

2) $\mathrm{SF}_{6}$ 电气设备自恢复绝缘放电故障可以用 $\mathrm{SO}_{2}$ 和 $\mathrm{H}_{2} \mathrm{~S}$ 的浓度比值 $\mathrm{C}_{\mathrm{SO} 2} / \mathrm{C}_{\mathrm{H} 2 \mathrm{~S}}>7$ 判据准确判断。 $\mathrm{SO}_{2}$ 和 $\mathrm{H}_{2} \mathrm{~S}$ 的浓度允许比较大，即使浓度达到 $100 \mu \mathrm{L} / \mathrm{L}$ ， 仍然不会对设备安全运行产生即时的危险，可以从容 地计划、安排检修。

3) 裸导体过热, 可以使用自恢复绝缘放电故障的 判据进行故障判断; 导体过热，如果涉及非自恢复绝 缘 (固体绝缘), 由于多数固体绝缘分解温度较低, 容 易产生 $\mathrm{H}_{2} \mathrm{~S}$ 等其他气体， $\mathrm{C}_{\mathrm{SO} 2} / \mathrm{C}_{\mathrm{H} 2 \mathrm{~S}}$ 的数值就不同，这 时可以用非自恢复绝缘放电故障判据。 
4) 对于断路器开断、闭合操作过程异常，出现拒动、 不能正常开断、闭合的操作, 属于设备操作异常。必须 测试 $\mathrm{SF}_{6}$ 气体分解产物 $\mathrm{SO}_{2}$ 和 $\mathrm{H}_{2} \mathrm{~S}$ 浓度,并查明原因。

5) 对于断路器开断、闭合额定短路开断电流以上 的操作，应及时测试 $\mathrm{SF}_{6}$ 气体分解产物 $\mathrm{SO}_{2}$ 和 $\mathrm{H}_{2} \mathrm{~S}$ 浓 度, 确认断路器内部无异常, 才能继续运行。

\section{REFERENCES}

[1] IEC 60480-2004, "Guidelines for the checking and treatment of sulfur hexafluoride (SF6) taken from electrical equipment and specification for its re-use", 2004.

[2] X. S. Zhuang, "The examination and judgment of SF6 electrical equipment discharge failure," Asia-Pacific Power and Energy Engineering Conference (APPEEC'09), 2009. 